\title{
Dynamic Reliability Analysis Method of Degraded Mechanical Components Based on Process Probability Density Function of Stress
}

\author{
Peng Gao ${ }^{1}$ and Liyang Xie ${ }^{2}$ \\ ${ }^{1}$ Department of Chemical Mechanical Engineering, Liaoning Shihua University, Liaoning 113001, China \\ ${ }^{2}$ Department of Mechanical Engineering and Automation, Northeastern University, Liaoning 110004, China \\ Correspondence should be addressed to Peng Gao; gaogaopeng@163.com
}

Received 15 August 2013; Revised 5 January 2014; Accepted 20 January 2014; Published 6 March 2014

Academic Editor: Dan Simon

Copyright (C) 2014 P. Gao and L. Xie. This is an open access article distributed under the Creative Commons Attribution License, which permits unrestricted use, distribution, and reproduction in any medium, provided the original work is properly cited.

It is necessary to develop dynamic reliability models when considering strength degradation of mechanical components. Instant probability density function (IPDF) of stress and process probability density function (PPDF) of stress, which are obtained via different statistical methods, are defined, respectively. In practical engineering, the probability density function (PDF) for the usage of mechanical components is mostly PPDF, such as the PDF acquired via the rain flow counting method. For the convenience of application, IPDF is always approximated by PPDF when using the existing dynamic reliability models. However, it may cause errors in the reliability calculation due to the approximation of IPDF by PPDF. Therefore, dynamic reliability models directly based on PPDF of stress are developed in this paper. Furthermore, the proposed models can be used for reliability assessment in the case of small amount of stress process samples by employing the fuzzy set theory. In addition, the mechanical components in solar array of satellites are chosen as representative examples to illustrate the proposed models. The results show that errors are caused because of the approximation of IPDF by PPDF and the proposed models are accurate in the reliability computation.

\section{Introduction}

With the high requirements for safe operation of mechanical products, reliability assessment of mechanical components has received more and more attention and great efforts have been made for developing reliability models [1-6]. As a matter of fact, the stress-strength interference (SSI) model is the most important approach in the reliability assessment of mechanical components [7-12]. However, the SSI model is essentially a static reliability model without the factor of time taken into account, which is only suitable to calculate the reliability at a fixed time instant.

Conventional dynamic reliability models are developed under the assumption that the probability density function (PDF) of stress (the PDF of stress is derived from the PDF of load via the geometrical parameters of components) at each time instant or each load application is known. However, it requires a large amount of samples to acquire the PDF of stress at each moment, which is unaffordable and impractical in practical engineering. An alternative approximate method for the PDF statistics of mechanical components, such as the well-known rain flow counting (RFC) method, is to record a stress history and then approximate PDF of stress at each time instant by the PDF obtained from the recorded stress process sample. For descriptive convenience, we define the PDF of stress at each moment (or at each application of random load) as the instant PDF (IPDF) and the PDF of stress obtained directly from a stress history sample as the process PDF (PPDF). From the definition, it can be learned that the PDF obtained by using the RFC method is essentially a PPDF.

As a matter of fact, it may cause errors in the reliability calculation to approximate IPDF by PPDF. This is because the information embedded in the stress history, which reflects the relationship between application times of different stress with different magnitude within a specified period of time, is lost in the approximation process. The reason and the influences 
of the errors caused by the approximation will be illustrated in detail later. In this paper, we will develop dynamic reliability models of mechanical components directly based on the PPDF of stress, which are more straightforward and meaningful in practical engineering. Moreover, the proposed reliability models can be used in the case where the amount of stress process samples is small.

In addition, due to the randomness of stress at each time instant, which is characterized by IPDF, the PPDF from a stress process sample may vary from the PPDF from another sample. To deal with the variation in the statistical parameters of PPDF from different samples, a large amount of stress process samples are needed. However, because of the limitation in the economics and time, the collected data could be imprecise. In this case, it is inaccurate to model the uncertainty of the statistical parameters of PPDF via probability theory. The proposed fuzzy dynamic reliability models consider both the impreciseness caused by incomplete or nonobtainable information and the uncertainty in the statistical parameters of PPDF by employing the fuzzy set theory.

\section{Dynamic Reliability Models of Mechanical Components Based on PPDF of Stress}

2.1. Reliability Models of Mechanical Components with respect to Load Application Times. When the stress process is complex, IPDF is irrelative with PPDF and it is incorrect to approximate IPDF by PPDF. Only in the case of ergodic stationary stress process, in which IPDF is constant, some relationship between IPDF and PPDF can be found. Therefore, in the following section, we will mathematically derive the relationship between IPDF and PPDF on the assumption of constant IPDF. Suppose that the total number of load application times is $n$. Denote the PPDF of stress by $f_{1}(\bullet)$. Then, the number of the application times of load, which yields the stress $s$, can be expressed as follows:

$$
n_{1}=n f_{1}(s) \Delta s
$$

Denote the IPDF of stress by $f_{2}(\bullet)$ and the probability that stress $s$ appears at each application of load by $P_{1}$. Then the relationship between $f_{2}(\bullet)$ and $P_{1}$ can be written as follows:

$$
P_{1}=f_{2}(s) \Delta s
$$

According to the theory of probability, the probability that $s_{1}$ appears for $n_{1}$ times within the $n$ times application of load can be expressed as follows:

$$
P_{2}=\left(\begin{array}{c}
n \\
n_{1}
\end{array}\right) P_{1}^{n_{1}}\left(1-P_{1}\right)^{n-n_{1}}
$$

where

$$
\left(\begin{array}{c}
n \\
n_{1}
\end{array}\right)=\frac{n !}{n_{1} !\left(n-n_{1}\right) !}
$$

In the case where $n$ and $n_{1}$ are deterministic, we can obtain the maximum value of $P_{2}$ by solving the equation as follows

$$
\frac{\mathrm{d} \ln P_{2}}{\mathrm{~d} P_{1}}=0
$$

Substituting (3) into (5) yields

$$
\frac{\mathrm{d}\left(\ln \left(\begin{array}{c}
n \\
n_{1}
\end{array}\right)+n_{1} \ln P_{1}+\left(n-n_{1}\right) \ln \left(1-P_{1}\right)\right)}{\mathrm{d} P_{1}}=0 .
$$

Equation (6) can be simplified as follows:

$$
n_{1}\left(1-P_{1}\right)-\left(n-n_{1}\right) P_{1}=0
$$

Solving (7) yields

$$
P_{1}=\frac{n_{1}}{n}=f_{1}(s) \Delta s
$$

From (8), it can be seen that

$$
f_{1}(s)=f_{2}(s)
$$

Therefore, it can be learned that the IPDF is identical with the PPDF with the maximum probability. Nevertheless, it should be noted that probability is less than one. For example, suppose that $n=2$ and $n_{1}=1$. From (8), it can be obtained that $P_{1}=0.5$. Substituting $P_{1}$ into the right-hand side of (3) yields that $P_{2}$ is equal to 0.5 rather than one. Therefore, in some cases, the approximation may cause errors in the reliability calculation, especially when developing dynamic reliability models where load application times or time is taken into account. To address this problem, reliability models with respect to load application times are proposed in this section, which are derived directly according to the statistical meaning of PPDF of stress. It should be noted that the dynamic reliability models based on PPDF are not limited by the format of PPDF distribution. When the PPDF $f_{t}(s)$ is obtained, the variation in the reliability of mechanical components can be evaluated by using the proposed reliability models.

As analyzed above, the PPDF of stress reflects the relationship between application times of different stress with different magnitude within a specified period of time. Provided that the total number of load application times is $n$, according to the statistical meaning of $f_{t}(\bullet)$, the application times of load, which yields stress $s$, can be expressed as follows:

$$
n_{i}=n f_{t}(s) \Delta s .
$$


Therefore, the reliability under the application of the random load for $n$ times can be calculated as follows:

$$
\begin{aligned}
R(n) & =\prod_{i}\left[\int_{s_{i}}^{\infty} f_{r}(r) d r\right]^{n f_{t}\left(s_{i}\right) \Delta s} \\
& =\exp \left\{n \int_{-\infty}^{\infty} f_{t}(s) \ln \left[\int_{s_{i}}^{\infty} f_{r}(r) d r\right] \mathrm{d} s\right\} .
\end{aligned}
$$

According to the Miner damage accumulation rule, strength degrades under multiple applications of random load, which should be modeled as the function of both the load application times and the magnitude of stress. The remaining strength at the $n$th application of load can be expressed as [13]

$$
r(n)=r_{0}[1-D(n-1)]^{a},
$$

where $r_{0}$ and $a$ are material parameters. $D(n)$ is cumulative damage that is determined by the application times and magnitude of stress. According to the $S-N$ curve formula, it can be obtained that

$$
s^{m} N_{s}=C,
$$

where $m$ and $C$ are material parameters and $N_{s}$ represents the lifetime of components under stress $s$. According to the damage equivalence rule, Miner damage accumulation rule, and the statistical meaning of PPDF, the remaining strength can be given by [14]

$$
r(n)=r_{0}\left[1-\frac{(n-1)}{C} \int_{-\infty}^{\infty} s^{m} f_{t}(s) \mathrm{d} s\right]^{a}
$$

When considering the randomness of the material parameter $C$ and the initial strength $r_{0}$, the reliability after the load application for $n$ times can be given by

$$
\begin{aligned}
R(n) & =\exp \left\{\int_{-\infty}^{\infty} f_{t}(s)\left[\sum_{k=1}^{n} \ln \left(\int_{-\infty}^{\infty} f_{C}(C)\left(1-\int_{-\infty}^{s C^{a} /\left(C-(k-1) \int_{-\infty}^{\infty} s^{m} f_{t}(s) \mathrm{d} s\right)^{a}} f_{r_{0}}\left(r_{0}\right) \mathrm{d} r_{0}\right) \mathrm{d} C\right)\right] \mathrm{d} s\right\} \\
& =\exp \left\{\int_{-\infty}^{\infty} f_{t}(s)\left[\sum_{k=1}^{n} \ln \left(1-\int_{-\infty}^{\infty} f_{C}(C) \int_{-\infty}^{s C^{a} /\left(C-(k-1) \int_{-\infty}^{\infty} s^{m} f_{t}(s) \mathrm{d} s\right)^{a}} f_{r_{0}}\left(r_{0}\right) \mathrm{d} r_{0} \mathrm{~d} C\right)\right] \mathrm{d} s\right\},
\end{aligned}
$$

where $f_{r_{0}}\left(r_{0}\right)$ and $f_{C}(C)$ are the PDF of the initial strength $r_{0}$ and the material parameter $C$, respectively. According to the definition of failure rate, the failure rate with respect to load application times can be written as

$$
\begin{aligned}
& h(n) \\
& =\frac{F(n+1)-F(n)}{R(n)} \\
& =\frac{R(n)-R(n+1)}{R(n)} \\
& =1-\exp \left\{\int _ { - \infty } ^ { \infty } f _ { t } ( s ) \operatorname { l n } \left(1-\int_{-\infty}^{\infty} f_{C}(C) \quad f_{r_{0}}^{s C^{a} /\left(C-n \int_{-\infty}^{\infty} s^{m} f_{t}(s) \mathrm{d} s\right)^{a}}\right.\right. \\
& \left.\left.\quad \times \int_{-\infty} \times\left(r_{0}\right) \mathrm{d} r_{0} \mathrm{~d} C\right) \mathrm{~d} s\right\} .
\end{aligned}
$$

Due to the limitation in the economics and time, the collected data could be imprecise. In this case, it is inaccurate to model the uncertainty of the statistical parameters of PPDF via probabilistic methods. The fuzzy reliability models provide a mathematical tool to deal with the impreciseness caused by incomplete or nonobtainable information. Therefore, fuzzy dynamic reliability models are proposed in this paper.

To make the best of the stress history samples for dynamic reliability analysis, stress is modeled as a variable characterized by its PPDF with fuzzy distribution parameters to deal with the variation or imprecision of stress distribution from different stress history samples due to insufficient experimental data. Denote the PDF of fuzzy distribution parameters $\mathbf{K}$ at the level of $\alpha$ by $f_{K \alpha}(\mathbf{K})$ and the PPDF of stress with the distribution parameters $\mathbf{K}$ by $f_{t \alpha}(s, \mathbf{K})$. Then, according to the Bayes law for continuous variables, the reliability of components at the level of $\alpha$ can be given by

$$
1
$$

$$
R_{\alpha}(n)=\int_{\mathbf{K}^{L}}^{\mathbf{K}^{U}} f_{K \alpha}(\mathbf{K}) \exp \left\{\int_{-\infty}^{\infty} f_{t \alpha}(s, \mathbf{K})\left[\sum_{k=1}^{n} \ln \left(1-\int_{-\infty}^{\infty} f_{C}(C) \int_{-\infty}^{s C^{a} /\left(C-(k-1) \int_{-\infty}^{\infty} s^{m} f_{t \alpha}(s, \mathbf{K}) \mathrm{d} s\right)^{a}} f_{r_{0}}\left(r_{0}\right) \mathrm{d} r_{0} \mathrm{~d} C\right)\right] \mathrm{d} s\right\} \mathrm{d} \mathbf{K}
$$


The failure rate with respect to load application times at the level of $\alpha$ can be written as

$$
\begin{aligned}
h(n)= & \frac{F(n+1)-F(n)}{R(n)} \\
= & \left\{\int_{\mathbf{K}^{L}}^{\mathbf{K}^{U}} f_{K \alpha}(\mathbf{K}) \exp \left\{\int_{-\infty}^{\infty} f_{t \alpha}(s, \mathbf{K})\left[\sum_{k=1}^{n} \ln \left(1-\int_{-\infty}^{\infty} f_{C}(C) \int_{-\infty}^{s C^{a} /\left(C-(k-1) \int_{-\infty}^{\infty} s^{m} f_{t \alpha}(s, \mathbf{K}) \mathrm{d} s\right)^{a}} f_{r_{0}}\left(r_{0}\right) \mathrm{d} r_{0} \mathrm{~d} C\right)\right] \mathrm{d} s\right\} \mathrm{d} \mathbf{K}\right] \\
& \left.-\int_{\mathbf{K}^{L}}^{\mathbf{K}^{U}} f_{K \alpha}(\mathbf{K}) \exp \left\{\int_{-\infty}^{\infty} f_{t \alpha}(s, \mathbf{K})\left[\sum_{k=1}^{n+1} \ln \left(1-\int_{-\infty}^{\infty} f_{C}(C) \int_{-\infty}^{s C^{a} /\left(C-(k-1) \int_{-\infty}^{\infty} s^{m} f_{t \alpha}(s, \mathbf{K}) \mathrm{d} s\right)^{a}} f_{r_{0}}\left(r_{0}\right) \mathrm{d} r_{0} \mathrm{~d} C\right)\right] \mathrm{d} s\right\} \mathrm{d} \mathbf{K}\right\} \\
& \times\left\{\int_{\mathbf{K}^{L}}^{\mathbf{K}^{U}} f_{K \alpha}(\mathbf{K}) \exp \left\{\int_{-\infty}^{\infty} f_{t \alpha}(s, \mathbf{K})\left[\sum_{k=1}^{n} \ln \left(1-\int_{-\infty}^{\infty} f_{C}(C) \int_{-\infty}^{s C^{a} /\left(C-(k-1) \int_{-\infty}^{\infty} s^{m} f_{t \alpha}(s, \mathbf{K}) \mathrm{d} s\right)^{a}} f_{r_{0}}\left(r_{0}\right) \mathrm{d} r_{0} \mathrm{~d} C\right)\right] \mathrm{d} s\right\} \mathrm{d} \mathbf{K}\right\} .
\end{aligned}
$$

Therefore, according to the decomposition theorem of fuzzy set, the reliability of components can be expressed as follows:

$$
R(n)=\int_{0}^{1} \int_{\mathbf{K}^{L}}^{\mathbf{K}^{U}} f_{K \alpha}(\mathbf{K}) \exp \left\{\int_{-\infty}^{\infty} f_{t \alpha}(s, \mathbf{K})\left[\sum_{k=1}^{n} \ln \left(1-\int_{-\infty}^{\infty} f_{C}(C) \int_{-\infty}^{s C^{a} /\left(C-(k-1) \int_{-\infty}^{\infty} s^{m} f_{t \alpha}(s, \mathbf{K}) d s\right)^{a}} f_{r_{0}}\left(r_{0}\right) \mathrm{d} r_{0} \mathrm{~d} C\right)\right] \mathrm{d} s\right\} \mathrm{d} \mathbf{K} \mathrm{d} \alpha
$$

$$
\begin{aligned}
h(n)= & \frac{F(n+1)-F(n)}{R(n)} \\
= & \left\{\int_{0}^{1} \int_{\mathbf{K}^{L}}^{\mathbf{K}^{U}} f_{K \alpha}(\mathbf{K}) \exp \left\{\int_{-\infty}^{\infty} f_{t \alpha}(s, \mathbf{K})\left[\sum_{k=1}^{n} \ln \left(1-\int_{-\infty}^{\infty} f_{C}(C) \int_{-\infty}^{M} f_{r_{0}}\left(r_{0}\right) \mathrm{d} r_{0} \mathrm{~d} C\right)\right] \mathrm{d} s\right\} \mathrm{d} \mathbf{K} \mathrm{d} \alpha\right. \\
& \left.-\int_{0}^{1} \int_{\mathbf{K}^{L}}^{\mathbf{K}^{U}} f_{K \alpha}(\mathbf{K}) \exp \left\{\int_{-\infty}^{\infty} f_{t \alpha}(s, \mathbf{K})\left[\sum_{k=1}^{n+1} \ln \left(1-\int_{-\infty}^{\infty} f_{C}(C) \int_{-\infty}^{M} f_{r_{0}}\left(r_{0}\right) \mathrm{d} r_{0} \mathrm{~d} C\right)\right] \mathrm{d} s\right\} \mathrm{d} \mathbf{K} \mathrm{d} \alpha\right\} \\
& \times\left\{\int_{0}^{1} \int_{\mathbf{K}^{L}}^{\mathbf{K}^{U}} f_{K \alpha}(\mathbf{K}) \exp \left\{\int_{-\infty}^{\infty} f_{t \alpha}(s, \mathbf{K})\left[\sum_{k=1}^{n} \ln \left(1-\int_{-\infty}^{\infty} f_{C}(C) \int_{-\infty}^{M} f_{r_{0}}\left(r_{0}\right) \mathrm{d} r_{0} \mathrm{~d} C\right)\right] \mathrm{d} s\right\} \mathrm{d} \mathbf{K} \mathrm{d} \alpha\right\},
\end{aligned}
$$

where

$$
M=\frac{s C^{a}}{\left(C-(k-1) \int_{-\infty}^{\infty} s^{m} f_{t \alpha}(s, \mathbf{K}) \mathrm{d} s\right)^{a}}
$$

2.2. Dynamic Reliability Models of Mechanical Components with respect to Time. As a matter of fact, the Poisson process
Correspondingly, the failure rate with respect to load application times can be written as has been proved to be an effective stochastic process to mathematically describe the load process, in which load application times or arrival time of load is a random variable. Denote the intensity of the Poisson process by $\lambda(t)$. According to the properties of the Poisson process, the stochastic differential equation can be obtained as follows: 
Mathematical Problems in Engineering

5

$$
\begin{aligned}
& R(t+\Delta t) \\
& =R(t)\left\{\sum_{x=0}^{\infty} P(n=x)\right. \\
& \times[1-\lambda(t) \Delta t+\lambda(t) \Delta t \\
& \left.\left.\times \exp \left(\int_{-\infty}^{\infty} f_{t}(s)\left(\ln \left(1-\int_{-\infty}^{\infty} f_{C}(C) \int_{-\infty}^{s C^{a} /\left(C-x \int_{-\infty}^{\infty} s^{m} f_{t}(s) \mathrm{d} s\right)^{a}} f_{r_{0}}\left(r_{0}\right) \mathrm{d} r_{0} \mathrm{~d} C\right)\right) \mathrm{d} s\right)\right]\right\} \\
& =R(t)\left\{1-\lambda(t) \Delta t \sum_{x=0}^{\infty} P(n=x)\left[1-\exp \left(\int_{-\infty}^{\infty} f_{t}(s)\left(\ln \left(1-\int_{-\infty}^{\infty} f_{C}(C) \int_{-\infty}^{s C^{a} /\left(C-x \int_{-\infty}^{\infty} s^{m} f_{t}(s) \mathrm{d} s\right)^{a}} f_{r_{0}}\left(r_{0}\right) \mathrm{d} r_{0} \mathrm{~d} C\right)\right) \mathrm{d} s\right)\right]\right\} \\
& =R(t)\{1-\lambda(t) \Delta t \\
& \times \sum_{x=0}^{\infty} \frac{\left(\int_{0}^{t} \lambda(t) \mathrm{d} t\right)^{x}}{x !} \exp \left(-\int_{0}^{t} \lambda(t) \mathrm{d} t\right) \\
& \left.\times\left[1-\exp \left(\int_{-\infty}^{\infty} f_{t}(s)\left(\ln \left(1-\int_{-\infty}^{\infty} f_{C}(C) \int_{-\infty}^{s C^{a} /\left(C-x \int_{-\infty}^{\infty} s^{m} f_{t}(s) \mathrm{d} s\right)^{a}} f_{r_{0}}\left(r_{0}\right) \mathrm{d} r_{0} \mathrm{~d} C\right)\right) \mathrm{d} s\right)\right]\right\} .
\end{aligned}
$$

Simplify (22) yields

$$
\begin{aligned}
& \frac{R(t+\Delta t)-R(t)}{R(t) \Delta t} \\
& =-\lambda(t) \exp \left(-\int_{0}^{t} \lambda(t) \mathrm{d} t\right) \\
& \quad \times \sum_{x=0}^{\infty} \frac{\left(\int_{0}^{t} \lambda(t) \mathrm{d} t\right)^{x}}{x !} \\
& \quad \times\left[1-\exp \left(\int_{-\infty}^{\infty} f_{t}(s)\left(\ln \left(1-\int_{-\infty}^{\infty} f_{C}(C) \int_{-\infty}^{s C^{a} /\left(C-x \int_{-\infty}^{\infty} s^{m} f_{t}(s) \mathrm{d} s\right)^{a}} f_{r_{0}}\left(r_{0}\right) \mathrm{d} r_{0} \mathrm{~d} C\right)\right) \mathrm{d} s\right)\right] .
\end{aligned}
$$

Let $\Delta t \rightarrow 0$. The above equation becomes

$$
\begin{aligned}
& \frac{d R(t)}{R(t) \mathrm{d} t} \\
& =-\lambda(t) \exp \left(-\int_{0}^{t} \lambda(t) \mathrm{d} t\right) \\
& \quad \times \sum_{x=0}^{\infty} \frac{\left(\int_{0}^{t} \lambda(t) \mathrm{d} t\right)^{x}}{x !} \\
& \quad \times\left[1-\exp \left(\int_{-\infty}^{\infty} f_{t}(s)\left(\ln \left(1-\int_{-\infty}^{\infty} f_{C}(C) \int_{-\infty}^{s C^{a} /\left(C-x \int_{-\infty}^{\infty} s^{m} f_{t}(s) \mathrm{d} s\right)^{a}} f_{r_{0}}\left(r_{0}\right) \mathrm{d} r_{0} \mathrm{~d} C\right)\right) \mathrm{d} s\right)\right] .
\end{aligned}
$$




$$
\begin{aligned}
R(t)=\exp \{- & \int_{0}^{t} \lambda(t) \exp \left(-\int_{0}^{t} \lambda(t) \mathrm{d} t\right) \\
& \times \sum_{x=0}^{\infty} \frac{\left(\int_{0}^{t} \lambda(t) \mathrm{d} t\right)^{x}}{x !} \\
& \left.\times\left[1-\exp \left(\int_{-\infty}^{\infty} f_{t}(s)\left(\ln \left(1-\int_{-\infty}^{\infty} f_{C}(C) \int_{-\infty}^{s C^{a} /\left(C-x \int_{-\infty}^{\infty} s^{m} f_{t}(s) \mathrm{d} s\right)^{a}} f_{r_{0}}\left(r_{0}\right) \mathrm{d} r_{0} \mathrm{~d} C\right)\right) \mathrm{d} s\right)\right] \mathrm{d} t\right\}
\end{aligned}
$$

From (25), the failure rate can be derived as follows:

$$
\begin{aligned}
& \beta(t)= \lambda(t) \exp \left(-\int_{0}^{t} \lambda(t) \mathrm{d} t\right) \\
& \times \sum_{x=0}^{\infty} \frac{\left(\int_{0}^{t} \lambda(t) \mathrm{d} t\right)^{x}}{x !} \\
& \times\left[1-\exp \left(\int_{-\infty}^{\infty} f_{t}(s)\left(\ln \left(1-\int_{-\infty}^{\infty} f_{C}(C) \int_{-\infty}^{s C^{a} /\left(C-x \int_{-\infty}^{\infty} s^{m} f_{t}(s) \mathrm{d} s\right)^{a}} f_{r_{0}}\left(r_{0}\right) \mathrm{d} r_{0} \mathrm{~d} C\right)\right) \mathrm{d} s\right)\right] .
\end{aligned}
$$

Analogical to the derivation process of (19), when considering the variation of the distribution parameters in the PPDF obtained from different stress history samples, the reliability of components at the level of $\alpha$ can be given by

$$
\begin{aligned}
& R_{\alpha}(t) \\
& \begin{aligned}
\int_{\mathbf{K}^{L}}^{\mathbf{K}^{U}} f_{K \alpha}(\mathbf{K}) \\
\quad \times \exp \left\{-\int_{0}^{t} \lambda(t) \exp \left(-\int_{0}^{t} \lambda(t) \mathrm{d} t\right)\right. \\
\quad \times \sum_{x=0}^{\infty} \frac{\left(\int_{0}^{t} \lambda(t) \mathrm{d} t\right)^{x}}{x !} \\
\left.\quad \times\left[1-\exp \left(\int_{-\infty}^{\infty} f_{t \alpha}(s, \mathbf{K})\left(\ln \left(1-\int_{-\infty}^{\infty} f_{C}(C) \int_{-\infty}^{s C^{a} /\left(C-x \int_{-\infty}^{\infty} s^{m} f_{t \alpha}(s, \mathbf{K}) \mathrm{d} s\right)^{a}} f_{r_{0}}\left(r_{0}\right) \mathrm{d} r_{0} \mathrm{~d} C\right)\right) \mathrm{d} s\right)\right] \mathrm{d} t\right\} \mathrm{d} \mathbf{K} .
\end{aligned}
\end{aligned}
$$


The failure rate at the level of $\alpha$ can be expressed as follows:

$$
\begin{aligned}
& \beta_{\alpha}(t) \\
& =\int_{\mathbf{K}^{L}}^{\mathbf{K}^{U}} f_{K \alpha}(\mathbf{K}) \lambda(t) \exp \left(-\int_{0}^{t} \lambda(t) \mathrm{d} t\right) \\
& \quad \times \sum_{x=0}^{\infty} \frac{\left(\int_{0}^{t} \lambda(t) \mathrm{d} t\right)^{x}}{x !} \\
& \quad \times\left[1-\exp \left(\int_{-\infty}^{\infty} f_{t \alpha}(s)\left(\ln \left(1-\int_{-\infty}^{\infty} f_{C}(C) \int_{-\infty}^{s C^{a} /\left(C-x \int_{-\infty}^{\infty} s^{m} f_{t \alpha}(s) \mathrm{d} s\right)^{a}} f_{r_{0}}\left(r_{0}\right) \mathrm{d} r_{0} \mathrm{~d} C\right)\right) \mathrm{d} s\right)\right] \mathrm{d} \mathbf{K}
\end{aligned}
$$

The reliability of mechanical components can be further derived as follows:

$$
\begin{aligned}
& R(t) \\
& =\int_{0}^{1} \int_{\mathbf{K}^{L}}^{\mathbf{K}^{U}} f_{K \alpha}(\mathbf{K}) \\
& \times \exp \left\{-\int_{0}^{t} \lambda(t) \exp \left(-\int_{0}^{t} \lambda(t) \mathrm{d} t\right)\right. \\
& \times \sum_{x=0}^{\infty} \frac{\left(\int_{0}^{t} \lambda(t) \mathrm{d} t\right)^{x}}{x !} \\
& \times\left[1-\exp \left(\int_{-\infty}^{\infty} f_{t \alpha}(s, \mathbf{K})\right.\right. \\
& \left.\left.\left.\times\left(\ln \left(1-\int_{-\infty}^{\infty} f_{C}(C) \int_{-\infty}^{s C^{a} /\left(C-x \int_{-\infty}^{\infty} s^{m} f_{t \alpha}(s, \mathbf{K}) \mathrm{d} s\right)^{a}} f_{r_{0}}\left(r_{0}\right) \mathrm{d} r_{0} \mathrm{~d} C\right)\right) \mathrm{d} s\right)\right] \mathrm{~d} t\right\} \mathrm{d} \mathbf{K} \mathrm{d} \alpha .
\end{aligned}
$$

$$
\begin{aligned}
& \beta(t) \\
& =\int_{0}^{1} \int_{\mathbf{K}^{L}}^{\mathbf{K}^{U}} f_{K \alpha}(\mathbf{K}) \lambda(t) \\
& \quad \times \exp \left(-\int_{0}^{t} \lambda(t) \mathrm{d} t\right) \\
& \quad \times \sum_{x=0}^{\infty} \frac{\left(\int_{0}^{t} \lambda(t) \mathrm{d} t\right)^{x}}{x !} \\
& \quad \times\left[1-\exp \left(\int_{-\infty}^{\infty} f_{t \alpha}(s)\left(\ln \left(1-\int_{-\infty}^{\infty} f_{C}(C) \int_{-\infty}^{s C^{a} /\left(C-x \int_{-\infty}^{\infty} s^{m} f_{t \alpha}(s) \mathrm{d} s\right)^{a}} f_{r_{0}}\left(r_{0}\right) \mathrm{d} r_{0} \mathrm{~d} C\right)\right) \mathrm{d} s\right)\right] \mathrm{d} \mathbf{K} \mathrm{d} \alpha .
\end{aligned}
$$


TABLE 1: Stress parameters and strength parameters.

\begin{tabular}{lccccc}
\hline$\mu\left(r_{0}\right)(\mathrm{MPa})$ & $\sigma\left(r_{0}\right)(\mathrm{MPa})$ & $\mu\left(s_{1}\right)(\mathrm{MPa})$ & $\sigma\left(s_{1}\right)(\mathrm{MPa})$ & $\mu\left(s_{2}\right)(\mathrm{MPa})$ & $\sigma\left(s_{2}\right)(\mathrm{MPa})$ \\
\hline 950 & 25 & 800 & 30 & 800 & 30 \\
\hline
\end{tabular}

TABLE 2: Stress parameters and material parameters.

\begin{tabular}{ccccccc}
\hline & $\mu(C)\left(\mathrm{MPa}^{2}\right)$ & $\sigma(C)\left(\mathrm{MPa}^{2}\right)$ & $\mu(s)(\mathrm{MPa})$ & $\sigma(s)(\mathrm{MPa})$ & $r_{0}(\mathrm{MPa})$ & $m$ \\
\hline$(1)$ & $8 \times 10^{9}$ & $10^{9}$ & 800 & 30 & 1000 & 2 \\
$(2)$ & $10^{10}$ & $10^{9}$ & 800 & 30 & 1000 & 1 \\
$(3)$ & $1.2 \times 10^{10}$ & $10^{9}$ & 800 & 30 & 1000 & 1 \\
\hline
\end{tabular}

TABLE 3: Stress parameters and material parameters.

\begin{tabular}{ccccccc}
\hline & $\mu(C)\left(\mathrm{MPa}^{2}\right)$ & $\sigma(C)\left(\mathrm{MPa}^{2}\right)$ & $\mu(s)(\mathrm{MPa})$ & $\sigma(s)(\mathrm{MPa})$ & $r_{0}(\mathrm{MPa})$ & $m$ \\
\hline$(1)$ & $10^{10}$ & $6 \times 10^{8}$ & 800 & 30 & 3000 & 1 \\
$(2)$ & $10^{10}$ & $8 \times 10^{8}$ & 800 & 30 & 30 & 1000 \\
$(3)$ & $10^{10}$ & $10^{9}$ & 800 & 30 & 1000 & 2 \\
$(4)$ & $10^{10}$ & $1.2 \times 10^{9}$ & 800 & 1000 & 1 \\
\hline
\end{tabular}

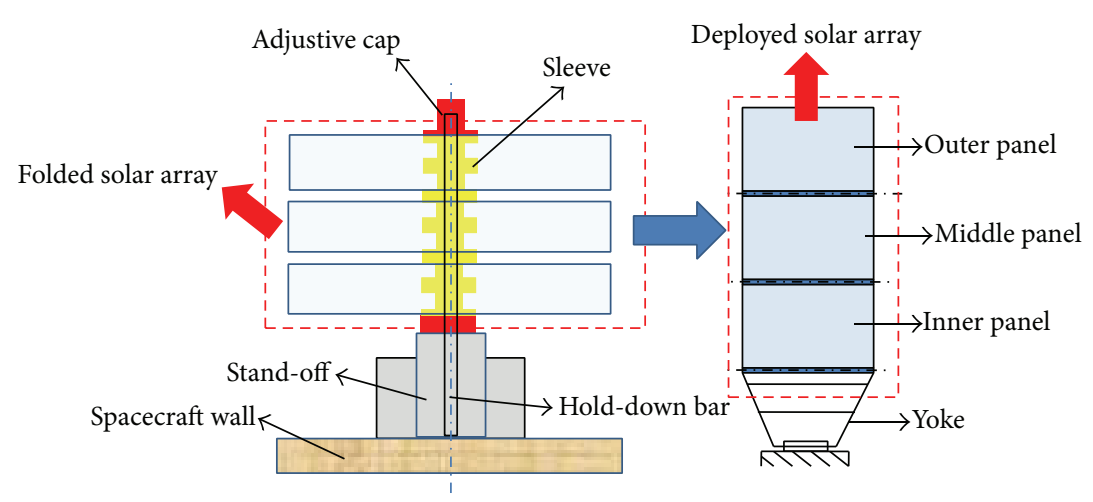

FIGURE 1: Structure of hold-down mechanism.

\section{Numerical Examples}

In numerical examples, we aim to validate the proposed dynamic reliability models, illustrate the errors caused by approximating IPDF by PPDF, and examine the influences of the statistical properties of material parameters on reliability. From the numerical examples, key factors that have great impacts on reliability could be identified and the large errors by the approximation of IPDF by PPDF will be represented.

The hold-down mechanism is an important mechanism in the solar array in the launch of satellites. The typical structure of hold-down mechanism is shown in Figure 1 [15]. In the hold-down mechanism, the maximum stress is applied to the hold-down bar. In this section, we will be focused on the reliability analysis of hold-down bar.

Case 1. Consider a hold-down bar operating under the application of random load. The stress and the strength follow the $s$-normal distribution with the distribution parameters listed in Table 1 . In Table $1, \mu(\bullet)$ and $\sigma(\bullet)$ represent the mean value and standard deviation of a variable, respectively. $\mu\left(s_{1}\right)$ and $\sigma\left(s_{1}\right)$ are the distribution parameters in the IPDF of stress, while $\mu\left(s_{2}\right)$ and $\sigma\left(s_{2}\right)$ are the distribution parameters in the PPDF of stress. The flow of the Monte Carlo simulation is shown in Figure 2. The reliability calculated by using IPDF of stress, the reliability calculated by using PPDF of stress, and the results from the Monte Carlo simulation are shown in Figure 3.

From Figure 3, it can be seen that the reliability calculated by using the method proposed in this paper shows good agreement with the results obtained from Monte Carlo simulations. In addition, the approximation of the IPDF of stress by the PPDF of stress may lead to errors in the reliability assessment.

Case 2. The distribution parameters of the material parameters are listed in Table 2. The reliability and failure rate are shown in Figures 4 and 5, respectively.

Case 3. The distribution parameters of the material parameters are listed in Table 3. The reliability and failure rate are shown in Figures 6 and 7, respectively.

From Figures 4 to 7 , it can be seen that the mean value of the material parameter $C$ has great influences on the 
TABLE 4: Stress parameters and material parameters.

\begin{tabular}{|c|c|c|c|c|c|c|c|c|c|}
\hline$\mu\left(s_{1}\right)(\mathrm{MPa})$ & $\sigma\left(s_{1}\right)(\mathrm{MPa})$ & $\mu\left(s_{2}\right)(\mathrm{MPa})$ & $\sigma\left(s_{2}\right)(\mathrm{MPa})$ & $\mu\left(r_{0}\right)(\mathrm{MPa})$ & $\sigma\left(r_{0}\right)(\mathrm{MPa})$ & $C\left(\mathrm{MPa}^{2}\right)$ & $\lambda\left(s^{-1}\right)$ & $m$ & $a$ \\
\hline 1000 & 30 & 1000 & 30 & 1200 & 20 & $10^{10}$ & 0.3 & 2 & 1 \\
\hline
\end{tabular}

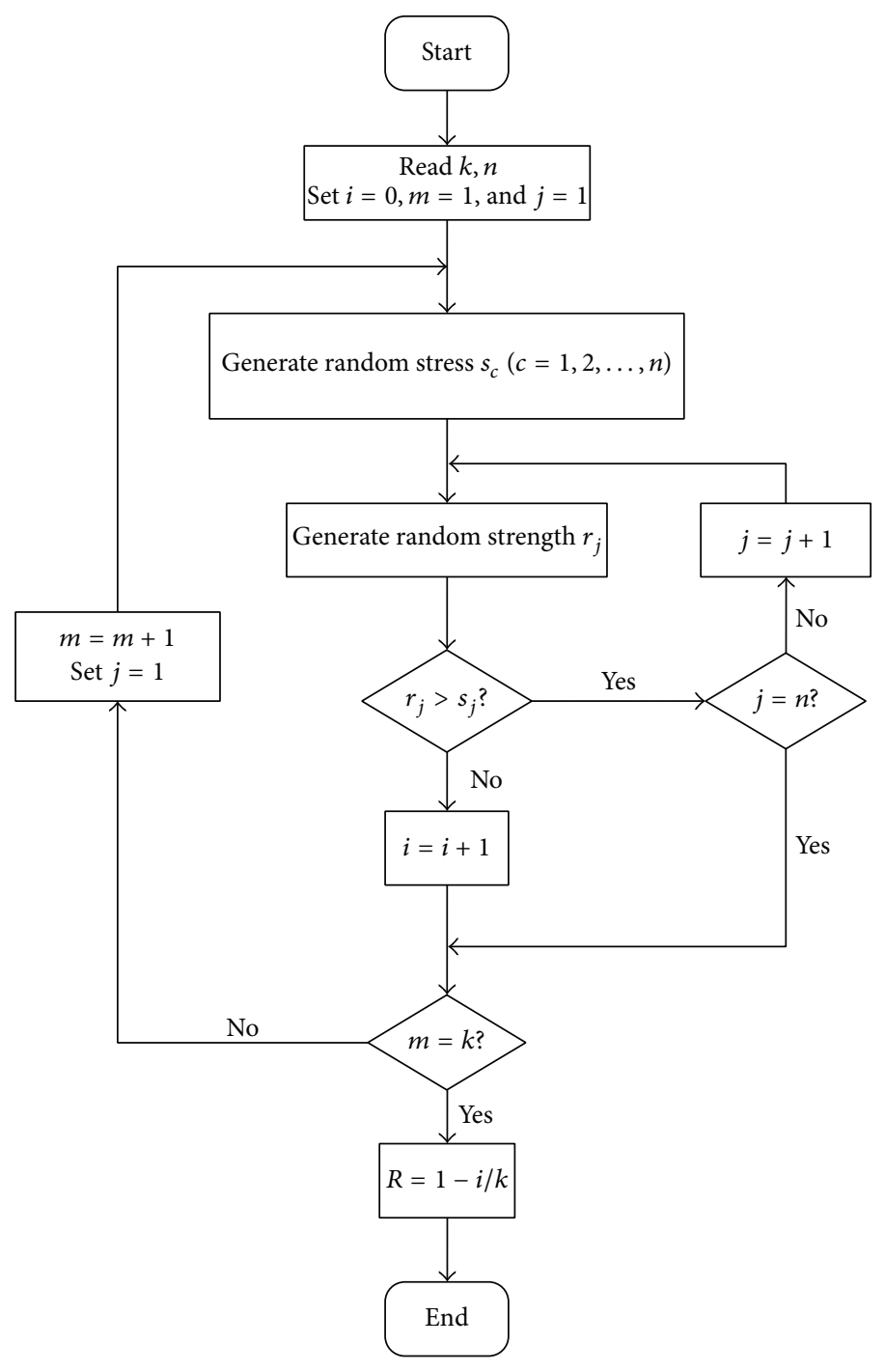

FIGURE 2: Flowchart of the Monte Carlo simulation.

reliability and failure rate of mechanical components. The reliability increases and the failure rate decreases rapidly with the increase in the mean value of the material parameter $C$. In addition, reliability decreases and failure rate increases with the increase in the dispersion of the material parameter $C$. Nevertheless, it should be noted that the reliability and failure rate are not sensitive to the dispersion of the material parameter $C$, even in the case of the existence of a large value of the standard deviation of the material parameter $C$. Meanwhile, the dispersion of initial strength has significant influences on the reliability and failure rate of mechanical components. Therefore, the dispersion of the material parameter $C$ can be neglected in the case where only an approximated reliability is required.
Case 4. The distribution parameters of the material parameters are listed in Table 4 . The reliability and failure rate are shown in Figures 8 and 9, respectively.

From Figures 8 and 9, it can be learned that reliability decreases and hazard rate increases with time. In the existing pieces literature, the dynamic reliability models for mechanical components are established on the assumption that the IPDF at each moment is known. In these models, the statistical parameters of stress and strength at each moment or each load application are given by assuming different format of stochastic process. However, in some cases, only a small amount of stress process samples can be obtained, which limits the usage of the reliability models based on IPDF to some extent. The proposed method based on PPDF 


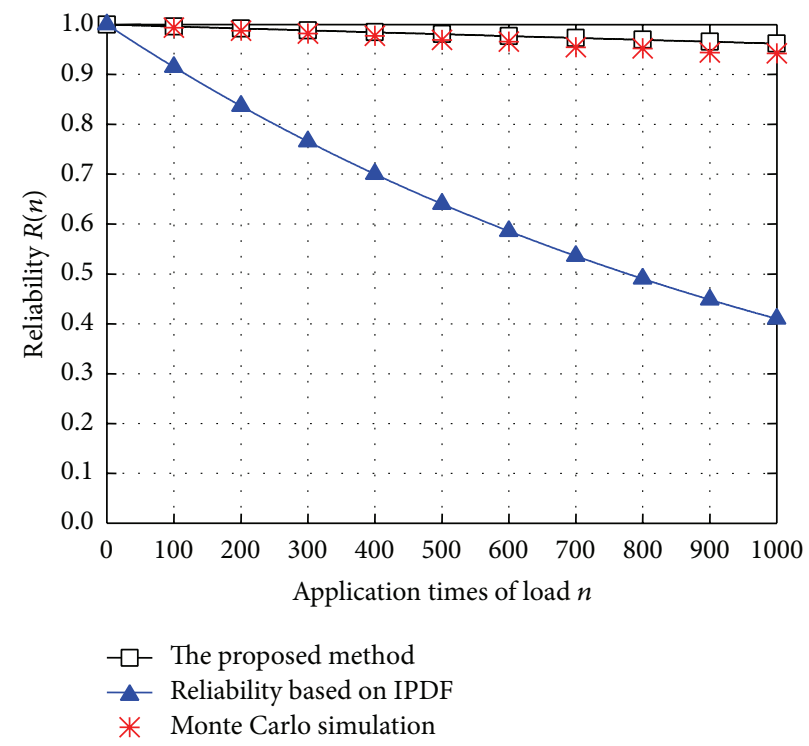

FIGURE 3: Comparison between the proposed method and Monte Carlo simulation.

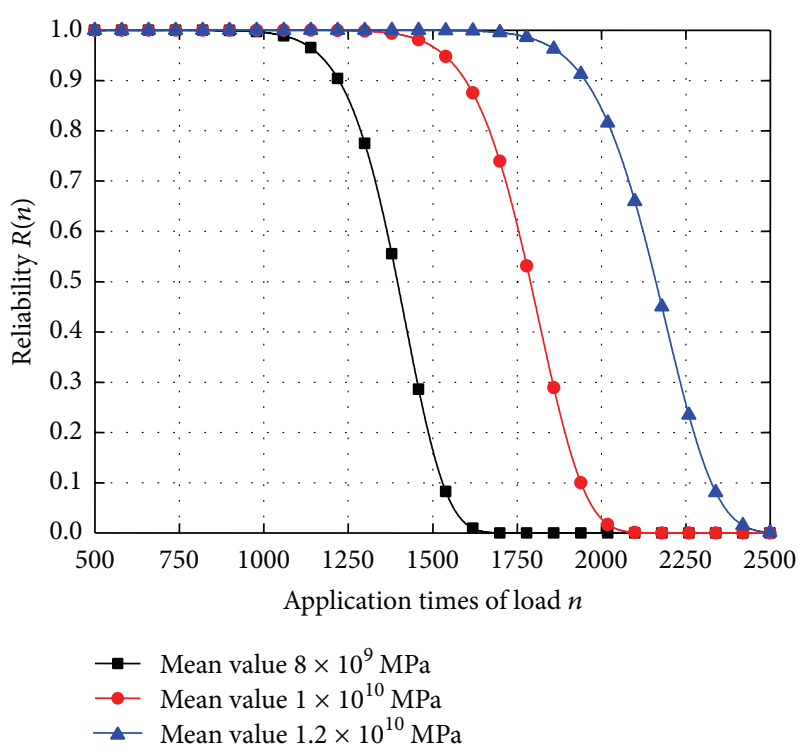

FIgURE 4: Reliability with different mean value of material parameter $C$.

provides an alternative choice for reliability assessment. The comparison between the results from the proposed reliability models based on the PPDF and the results based on the IPDF points out the error to approximate IPDF by PPDF.

\section{Conclusion}

It is imperative to develop dynamic reliability models, because strength degradation of mechanical components always occurs in the failure mode such as corrosion, wear, and erosion creep. In practice, the PDF for mechanical components is mostly PPDF. For the convenience of using

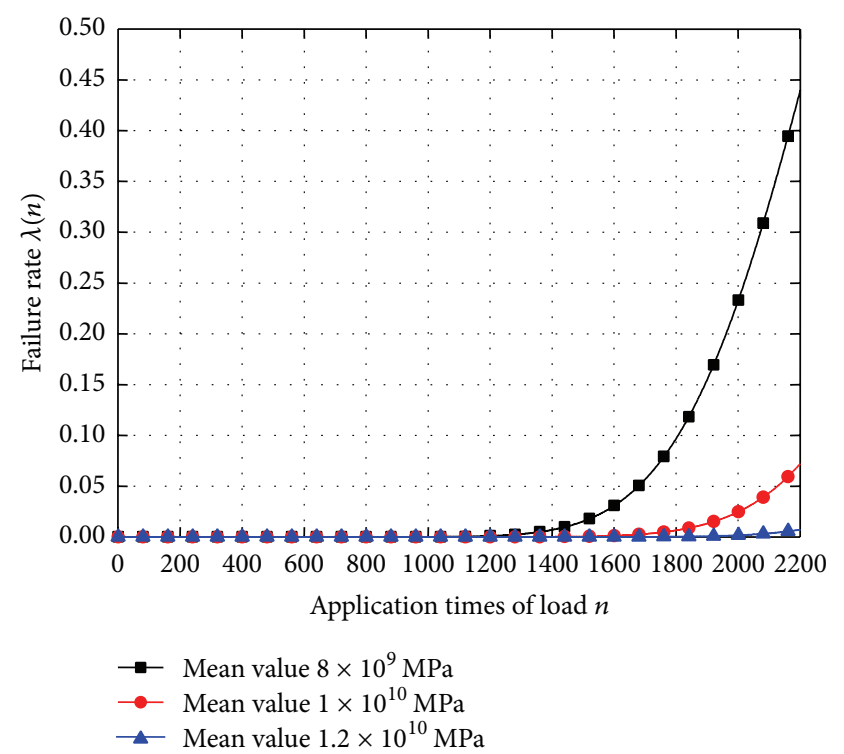

FIGURE 5: Failure rate with different mean value of material parameter $C$.

the existing dynamic reliability models, IPDF is always approximated by PPDF, which could cause large errors in the reliability calculation. The reliability models in this paper are established directly based on PPDF, which is more accurate than the result by using approximate method. Besides, due to the employment of fuzzy set theory, the proposed models can be used in the reliability estimation in the case of small amount of stress process samples. In addition, the proposed models can be used to analyze the influences of the variation in the statistical parameters of material parameters on the reliability and failure rate of mechanical components. The results show that the dispersion of material parameter $C$ 


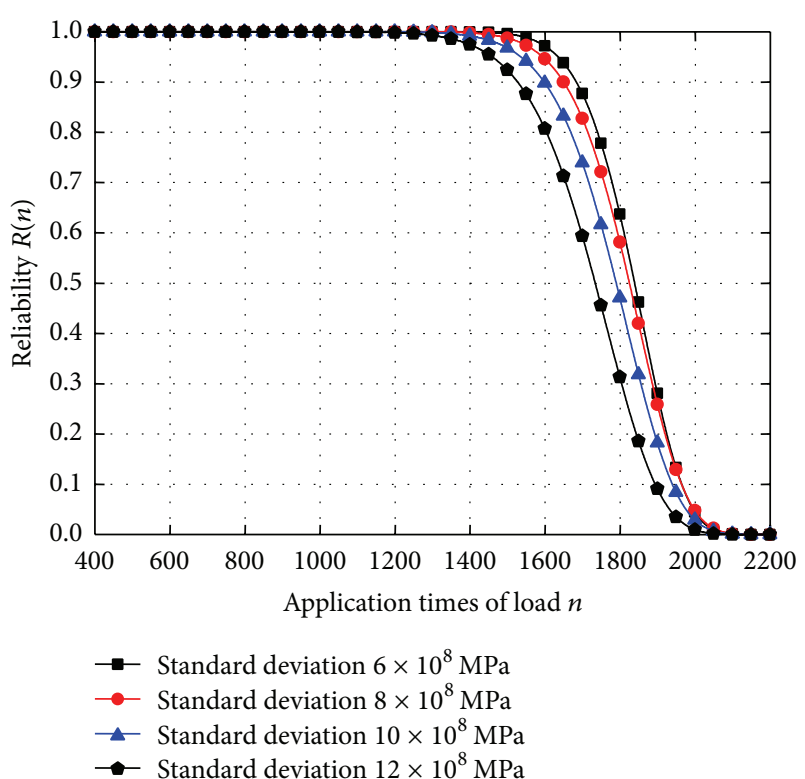

FIGURE 6: Reliability with different dispersion of material parameter C.

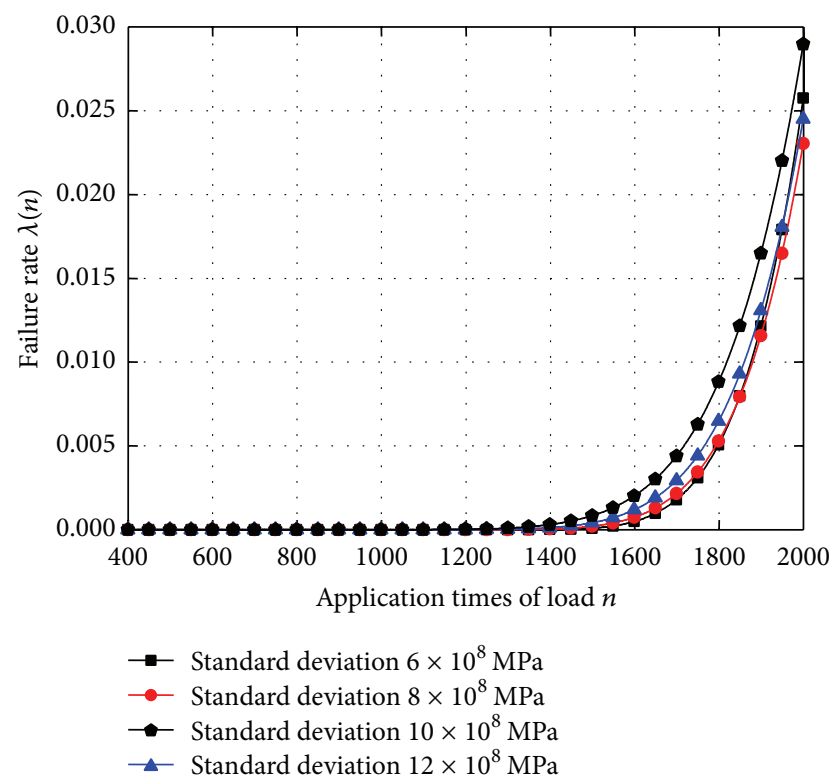

FIGURE 7: Failure rate with different dispersion of material parameter $C$.

has little impact on reliability and failure rate of mechanical components.

\section{Conflict of Interests}

The authors declare that there is no conflict of interests regarding the publication of this paper.

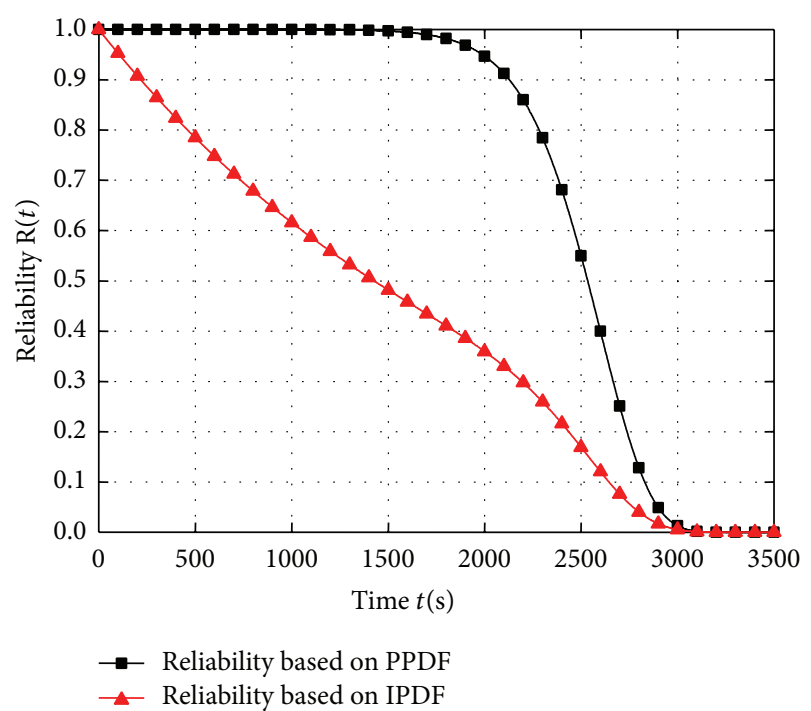

FIGURE 8: Reliability based on different PDF of stress.

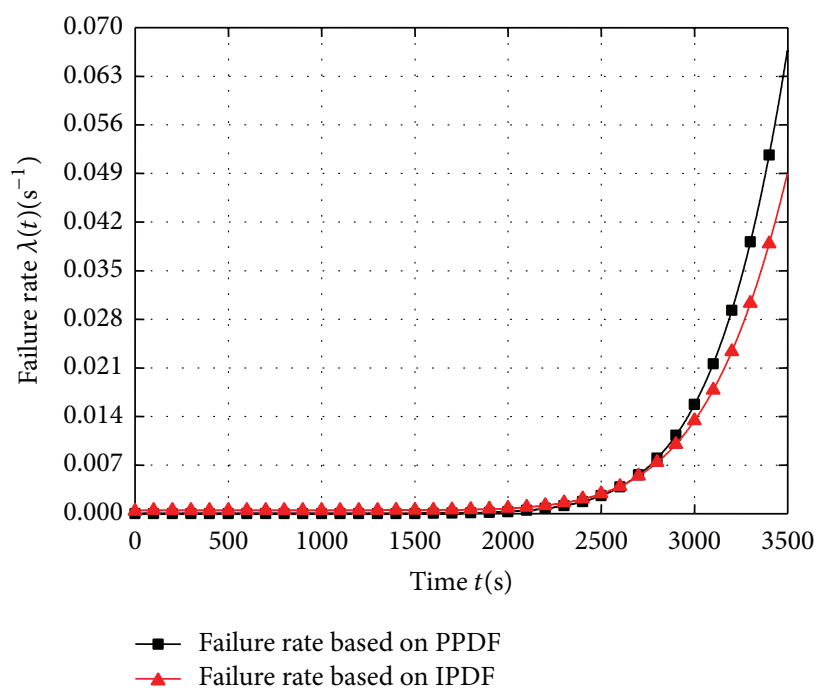

FIGURE 9: Failure rate based on different PDF of stress.

\section{Acknowledgments}

This work was supported by the Natural Science Foundation of China under Contract no. 51175240 and the National Science Foundation of China under Contract no. 11072123.

\section{References}

[1] L. Xie, J. Zhou, and C. Hao, "System-level load-strength interference based reliability modeling of $k$-out-of- $n$ system," Reliability Engineering and System Safety, vol. 84, no. 3, pp. 311-317, 2004.

[2] K. Shen, "An empirical approach to obtaining bounds on the failure probability through stress/strength interference," Reliability Engineering and System Safety, vol. 36, no. 1, pp. 7984, 1992.

[3] C. Q. Li, "Probability of plastic collapse of a structural system under nonstationary load processes," Computers and Structures, vol. 52, no. 1, pp. 69-78, 1994. 
[4] Y. Li and Q. Xu, "Stiffness analysis for a 3-PUU parallel kinematic machine," Mechanism and Machine Theory, vol. 43, no. 2, pp. 186-200, 2008.

[5] J. Wu, S. Yan, L. Xie, and P. Gao, "Reliability apportionment approach for spacecraft solar array using fuzzy reasoning Petri net and fuzzy comprehensive evaluation," Acta Astronautica, vol. 76, pp. 136-144, 2012.

[6] V. A. R. Camara and C. P. Tsokos, "The effect of loss functions on empirical bayes reliability analysis," Mathematical Problems in Engineering, vol. 4, no. 6, pp. 539-560, 1999.

[7] J. M. van Noortwijk, J. A. M. van der Weide, M. J. Kallen, and M. D. Pandey, "Gamma processes and peaks-over-threshold distributions for time-dependent reliability," Reliability Engineering and System Safety, vol. 92, no. 12, pp. 1651-1658, 2007.

[8] E. E. Lewis, "A load-capacity interference model for commonmode failures in 1-out-of-2: G systems," IEEE Transactions on Reliability, vol. 50, no. 1, pp. 47-51, 2001.

[9] W. Huang and R. G. Askin, "A generalized SSI reliability model considering stochastic loading and strength aging degradation," IEEE Transactions on Reliability, vol. 53, no. 1, pp. 77-82, 2004.

[10] V. Geidl and S. Saunders, "Calculation of reliability for timevarying loads and resistances," Structural Safety, vol. 4, no. 4, pp. 285-292, 1987.

[11] V. Rathod, O. P. Yadav, A. Rathore, and R. Jain, "Probabilistic modeling of fatigue damage accumulation for reliability prediction," International Journal of Quality, Statistics, and Reliability, vol. 2011, Article ID 718901, 10 pages, 2011.

[12] P. Wang and D. W. Coit, "Reliability prediction based on degradation modeling for systems with multiple degradation measures," in Proceedings of the Annual Reliability and Maintainability Symposium (RAMS '04), pp. 302-307, January 2004.

[13] Y.-W. Gu, W.-G. An, and H. An, "Structural reliability analysis under dead load and fatigue load," Acta Armamentarii, vol. 28, no. 12, pp. 1473-1477, 2007.

[14] P. Gao, S. Yan, L. Xie, and J. Wu, "Dynamic reliability analysis of mechanical components based on equivalent strength degradation paths," Journal of Mechanical Engineering, vol. 59, no. 6, pp. 387-399, 2013.

[15] J. J. Yuan, Design and Analysis of Satellite Structures, China Astronautic Publishing House, Beijing, China, 1st edition, 2004. 


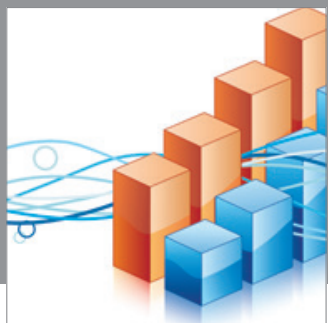

Advances in

Operations Research

mansans

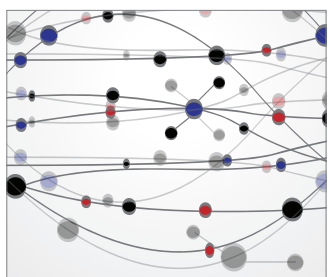

The Scientific World Journal
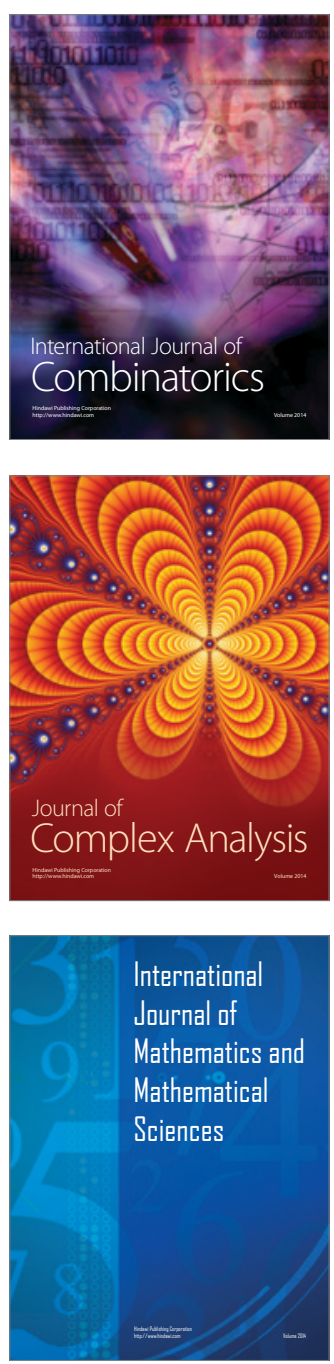
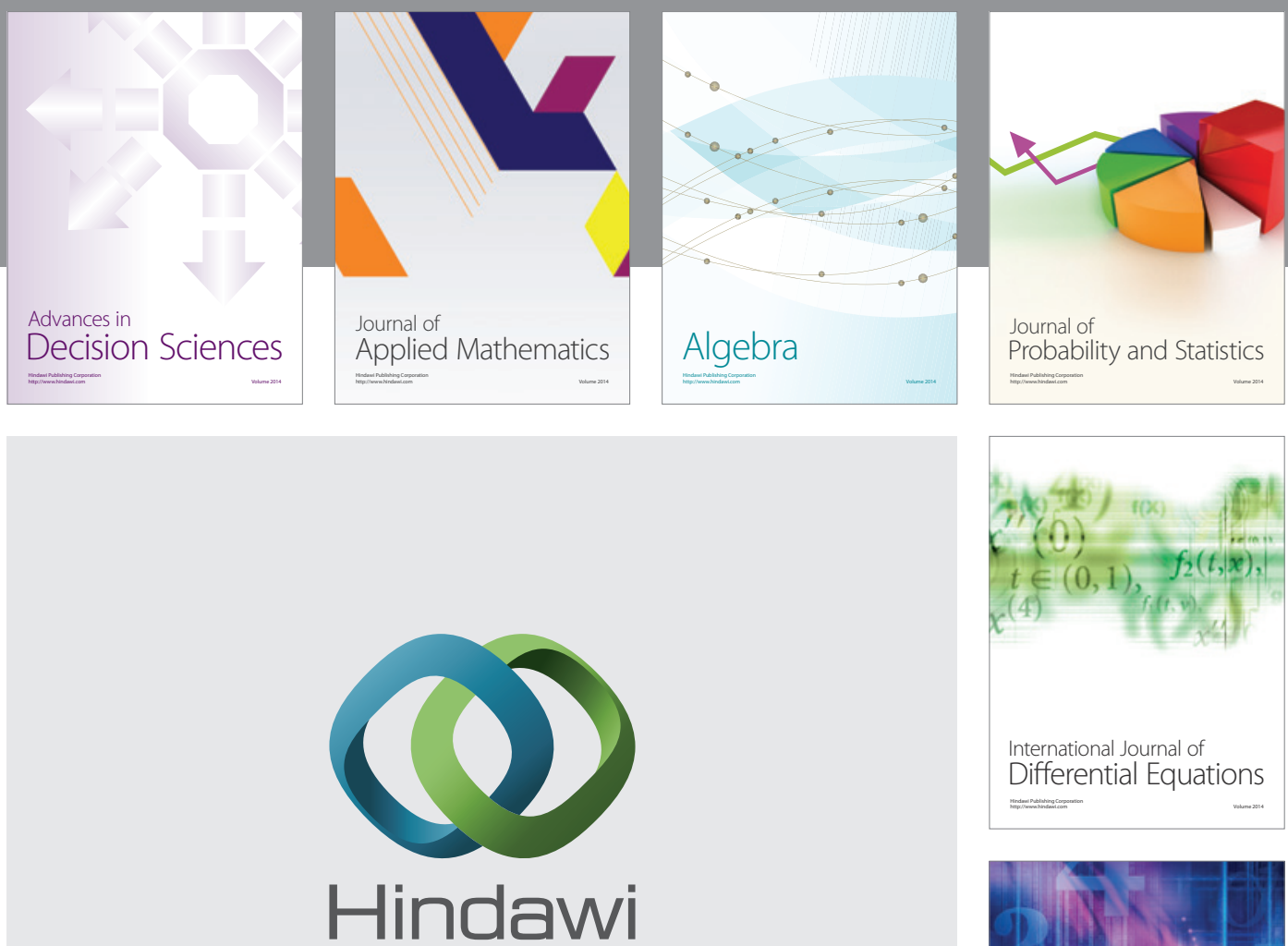

Submit your manuscripts at http://www.hindawi.com
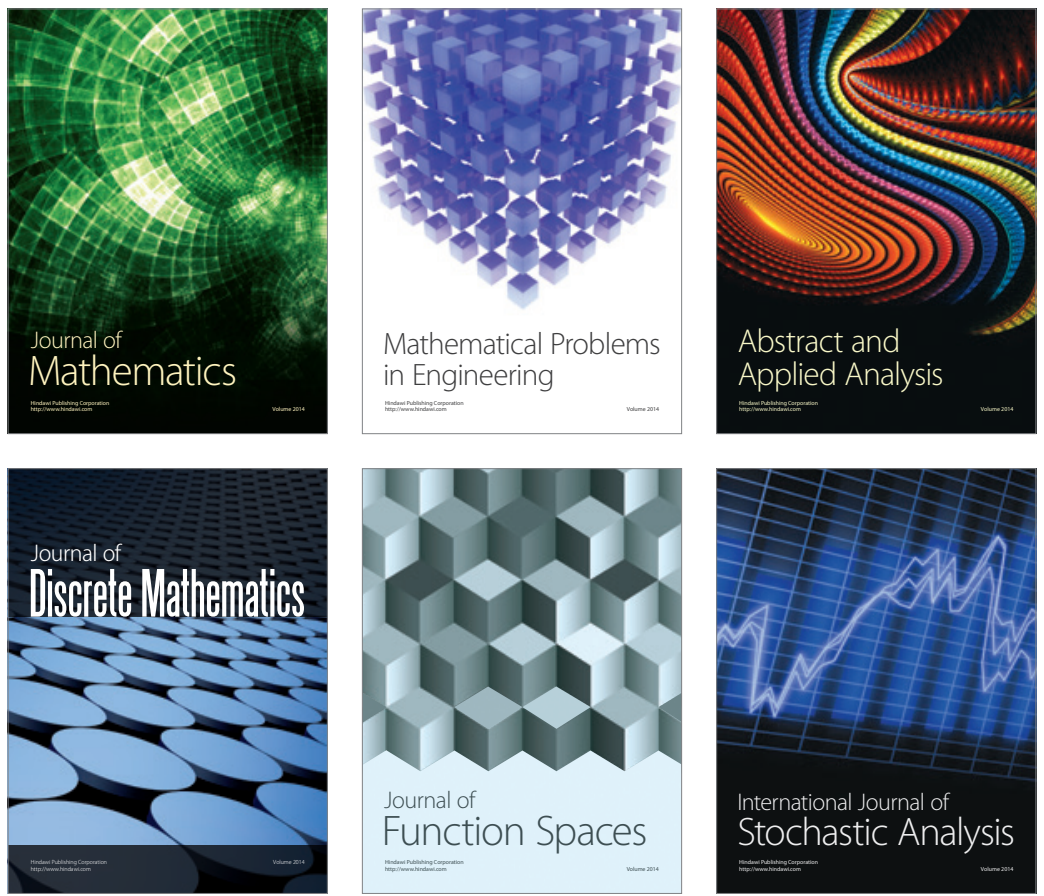

Journal of

Function Spaces

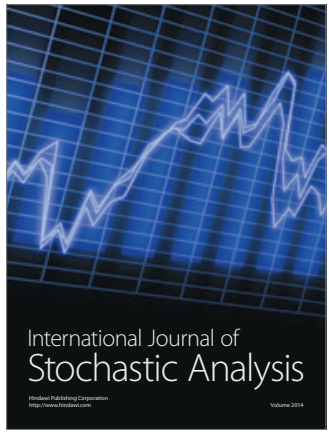

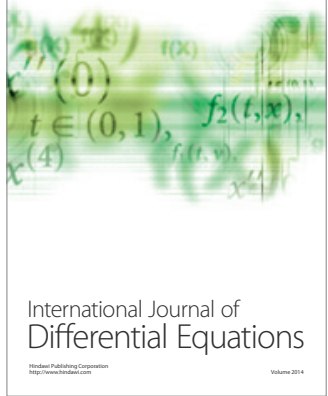
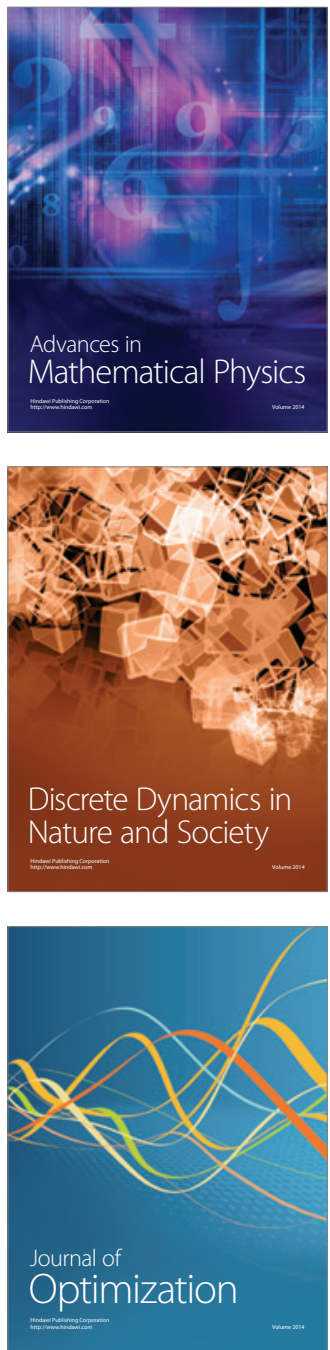